\title{
RF Power Amplifier Linearization in Professional Mobile Radio Communications Using Artificial Neural Networks
}

\author{
Raúl Gracia Sáez and Nicolás Medrano Marqués, Member, IEEE
}

\begin{abstract}
This paper is focused on the linearization of the radio frequency power amplifier of a professional digital handheld by means of an artificial neural network.

The simplicity of the neural network that is used, together with the fact that a feedback path is unnecessary, make this solution ideal to reduce both the cost of a handheld and its hardware complexity, while fully maintaining its performance.

A compensation system is also needed to keep the linearization characteristics of the neural network stable against frequency, temperature and voltage variations.

The whole solution that comprises both the neural network and the compensation system has been implemented in the DSP of a real handheld and afterwards fully tested. It has proved to be satisfactory to meet the telecommunication standard requirements in all frequency, temperature and voltage ranges under consideration, while efficient to lower the computational cost of the handheld and to make its internal hardware simpler in comparison with other traditional linearization techniques.

The results obtained demonstrate that a neural network can be used to linearize the power amplifiers that are used in transmitters of telecommunication equipment, leading to a significant reduction of both their hardware cost and complexity.
\end{abstract}

Index terms - Linearization, neural network, power amplifier, predistortion, radio frequency.

\section{INTRODUCTION}

In order to increase efficiency and to meet the telecommunications standards, many different techniques have been proposed and applied to extend the linear range of the power amplifier response: Cartesian Feedback [1], Feedforward [2], Volterra series [3], Memory Polynomials [4], Wiener and Hammerstein models [5], Lookup Tables (LUT) [6], Artificial Neural Networks (ANN) [7]-[13], Neural-Fuzzy systems [14], Genetic Algorithms [15], etc.

One of these telecommunications standards is TETRA. Terrestrial Trunked Radio (TETRA) is a professional communications standard designed for use in emergency services, public safety and transport services. Main characteristics of TETRA terminals are low cost, reliability and energy efficiency.

For this purpose, the most commonly used linearization method in TETRA is the Cartesian Feedback technique [1] (Figure 1). In this technique the output of the power amplifier

Manuscript received June 12, 2017; accepted May 14, 2018.

Raúl Gracia is with the R\&D department of the company Teltronic, Zaragoza, Spain (r-gracia@teltronic.es).

Nicolás Medrano is with the Faculty of Physics, University of Zaragoza, Zaragoza, Spain (nmedrano@unizar.es). is sampled and compared to the input, by applying both signals to an error amplifier. The need of a feedback path, the complexity of the Cartesian feedback algorithm and the wide printed circuit board area required to implement this solution, difficult its electronic realization in low-cost compact devices. Some commercial Application Specific Integrated Circuits (ASIC) integrate the Cartesian Feedback Loop and simplify the design, but greatly increasing the price and making the design of the equipment fully dependent of the life cycle of the ASIC, this is: there are not pin to pin alternatives and therefore the design is not protected against unexpected obsolescence or price fluctuations of the ASIC. Despite all the Cartesian method is still the only method used to linearize amplifiers in handhelds, because the other methods proposed until today increase the complexity of the system since they are not designed to be implemented in this type of low cost terminals.

In order to reduce those drawbacks of the Cartesian method, we propose the introduction of an Artificial Neural Network (ANN) [16] to determine the predistortion to be added to the input of the amplifier, therefore compensating the non-linear distortion introduced by the radio frequency (RF) amplifier (Figure 2), hence reducing the corresponding Adjacent Channel Power (ACP). The ACP is the frequency spectrum of the signal generated in a channel that interferes into the adjacent frequency channels [17]. The reduction of the ACP allows transmitting at higher RF power levels but using the same amplifier, therefore increasing its efficiency.

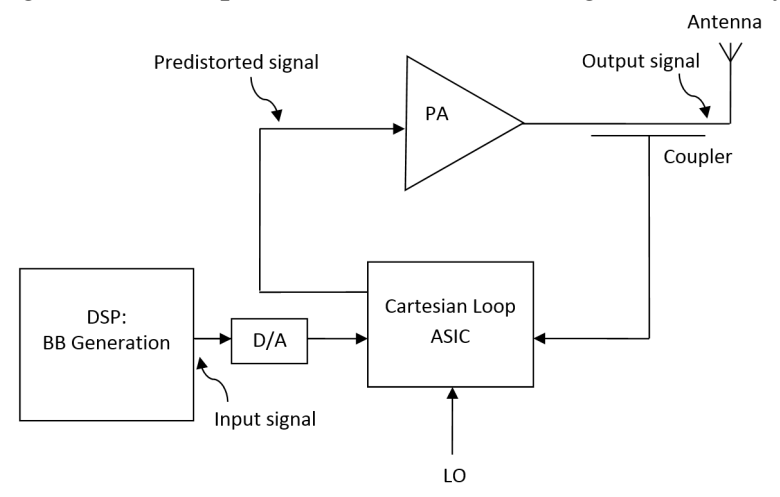

Figure 1. Linearization using Cartesian Feedback.

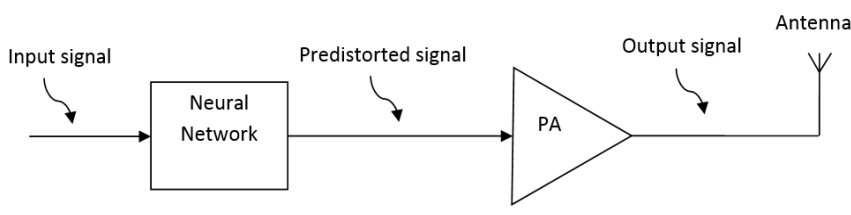

Figure 2. Basis of Neural Network Linearization. 
The introduction of the ANN will simplify the hardware and will reduce the cost of a commercial Tetra terminal. For this, it is essential to obtain a neural network architecture as simple as possible, so that it can be programmed into the DSP of the terminal, without the need of additional hardware.

Usually, the predistortion methods applied to linearize a power amplifier [2]-[15] require high computing resources, thus demanding the use of additional hardware computing devices as field-programmable gate arrays (FPGA), or at least, much more powerful DSPs to allow processing in the shortest time possible (fewer clock cycles possible). These solutions can be easily implemented in expensive telecommunication infrastructures, which present much larger space and energy resources available than a portable terminal. But, the use of these solutions in terminals, highly would increase its cost, size and energy requirements, thus jeopardizing its commercialization.

For this, the pre-distortion technique to be implemented into a portable terminal must be efficient and computationally simple enough to be implemented in the low cost DSPs available in those terminals, without additional hardware requirements. The currently available methods for linearizing RF power amplifiers in infrastructures and terminals are shown in Figure 3, including the neural network method for terminals proposed in this work.

\section{Methods for linearizing RF power amplifiers:}
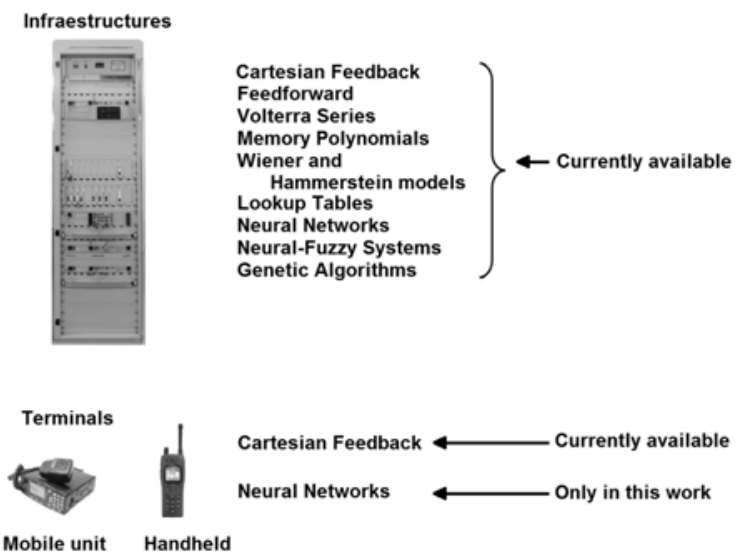

Figure 3. Methods for linearizing RF amplifiers in infrastructures and terminals.

The aim of this work is to design a low-cost, compact, simple and efficient ANN-based power linearization system, that can be introduced in the DSP of an ultra low-cost TETRA handheld powered by a single $3.7 \mathrm{~V}$ lithium battery, capable of transmitting $1 \mathrm{~W}$ of RF power in the $806-870 \mathrm{MHz}$ band and able to meet the TETRA standard requirements [18] in all the frequency, supply voltage and temperature working ranges.

This paper is structured as follows: Section II describes a baseline specification of the selected amplifier; Section III explains all considerations that were taken into account before the neural network was developed; section IV presents the results that were obtained for an ideal configuration of the neural network without changes in the external working conditions; Section V shows the compensation techniques applied for variations in the external working conditions (frequency, temperature and voltage), in order to guarantee the linearity under any condition; Section VI focuses on the implementation of the proposed technique in the low-cost DSP available; finally, Section VII presents the conclusions of this work.

\section{BASELINE SPECIFICATION}

For this work a commercial TETRA handheld that operates in the $806 \mathrm{MHz}-870 \mathrm{MHz}$ band has been selected. It has a classical 7.4 V transistor (PD54003L-E from ST Microelectronics) which works as a power amplifier and is supplied with two $3.7 \mathrm{~V}$ lithium cells. In order to reduce the cost of the handheld, that transistor has been replaced by a RF5110G RF amplifier from RFMD [19], whose voltage supply range is 2.7-4.8 V. Therefore, it can be powered by a single lithium cell. This amplifier has a Power Output Compression Point (P1dB), of about $31.5 \mathrm{dBm}$. The P1dB is the output power that generates a gain compression of $1 \mathrm{~dB}$ in the amplifier [17]. As the output power of the amplifier approaches P1dB, the amplifier is entering in the non-linear region of operation.

This amplifier has a maximum gain of $34 \mathrm{~dB}$ that can be limited by controlling the voltage value at the power control input pins (Vcontrol). It makes this amplifier a versatile selection suitable to minimize the cost of the handheld. Table I shows a comparison between the classical $7.4 \mathrm{~V}$ transistor PD54003L-E and the selected RF5110GDS amplifier.

TABLE I.

COMPARATIVE BETWEEN A CLASSICAL 7.4V TRANSISTOR AND THE SELECTED RF5110GDS AMPLIFIER.

\begin{tabular}{|c|c|c|}
\hline & RF5110GDS & PD54003L-E \\
\hline Gain & $34 \mathrm{~dB}$ & $18 \mathrm{~dB}$ \\
\hline Variable gain control & Yes & No \\
\hline P1dB & $31.5 \mathrm{dBm}$ & $32.5 \mathrm{dBm}$ \\
\hline Vcc & $2.7-4.8 \mathrm{~V}$ & $5-10 \mathrm{~V}$ \\
\hline Eff @ Pout=1W & $35 \%$ & $30 \%$ \\
\hline Package & $3 \times 3 \mathrm{~mm}$ & $5 \times 5 \mathrm{~mm}$ \\
\hline
\end{tabular}

Figures 4 and 5 show respectively the experimental AMAM and AM-PM characteristics [17] of the RF5110G power amplifier, acquired with an Agilent E5061B ENA Network Analyzer.

The ACP can also be characterized when the amplifier is excited with a TETRA standard signal to transmit an output power of $1 \mathrm{~W}$. Under these conditions, an ACP of $44 \mathrm{dBc}$ can be achieved at the output (Figure 6). Measurements were performed using an Agilent N9030A PXA Signal Analyzer.

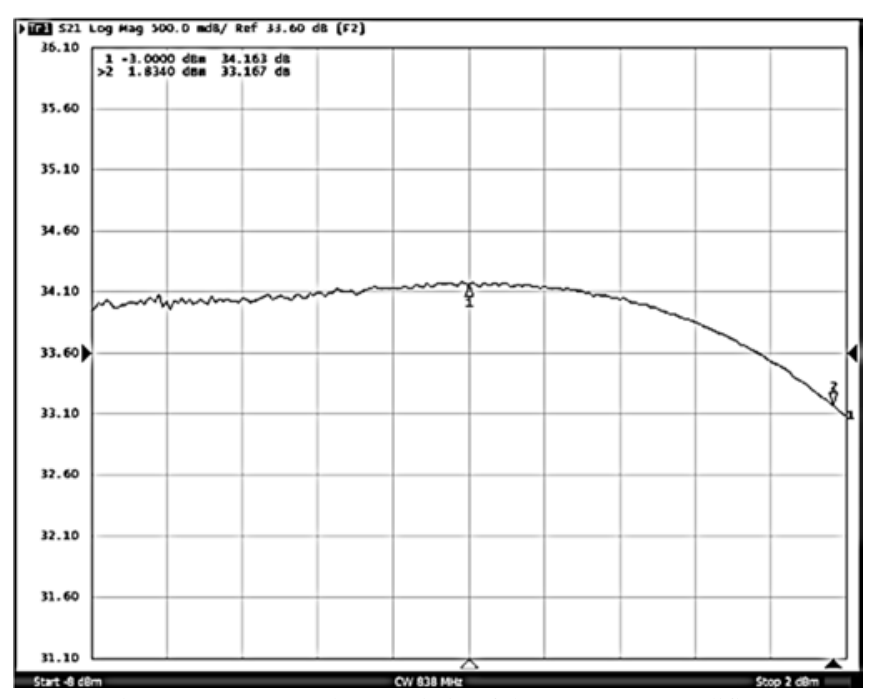

Figure 4. AM-AM characteristic of the RF5110G amplifier. 




Figure 5. AM-PM characteristic of the RF5110G amplifier.

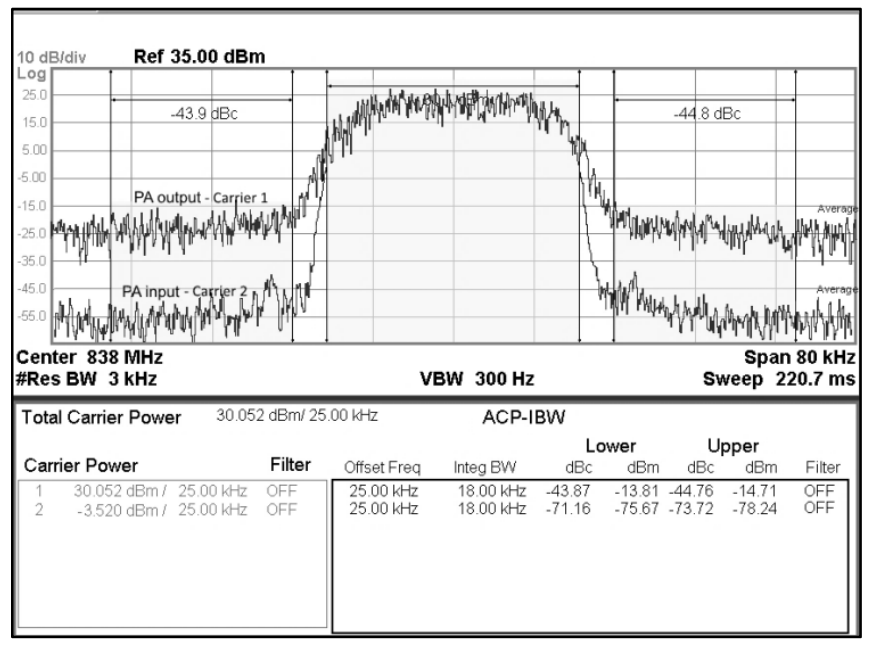

Figure 6. TETRA ACP of the RF5110G without previous linearization.

The selected amplifier model has been exposed to a process of early aging, obtaining that the gain and the $\mathrm{P} 1 \mathrm{~dB}$ of the amplifier keep constant. Thus, the non-linearity function maintains and therefore it can be assumed that the aging is not going to deteriorate the correction applied by the Neural Network.

\section{NEURAL NETWORK IMPLEMENTATION CONSIDERATIONS}

The process to obtain a suitable neural network, requires the next steps:

1. Collecting data sets for the training and test.

2. Configure the neural network (number of neurons and layers, training functions, transfer functions, performance estimation functions).

3. Train the network minimizing the training error.

4. Verify generalization ability.

\section{A. Data collection for training and test sets}

One of the most important considerations dealing with neural networks, is the acquisition of a suitable set of data for their training and test.

Figure 7 shows the acquisition system developed to get a suitable set of experimental data to be used in the linearization system. An Agilent ESG E4438C Vector Signal Generator provides the RF input signal to the amplifier according to the IQ signals previously defined using Matlab. The TETRA Output signal of the amplifier is demodulated using an Agilent N9030A PXA Signal Analyzer, obtaining the corresponding I and Q vectors that will be compared to the input IQ signals:

$$
o(t)=I(t) * \cos \left(\varpi_{c} t\right)-Q(t) * \sin \left(\varpi_{c} t\right)
$$

Where $o(t)$ is the RF signal, $I(t)$ and $Q(t)$ are the phase and quadrature baseband components, and $\omega_{c}$ is the angular frequency of the RF carrier.

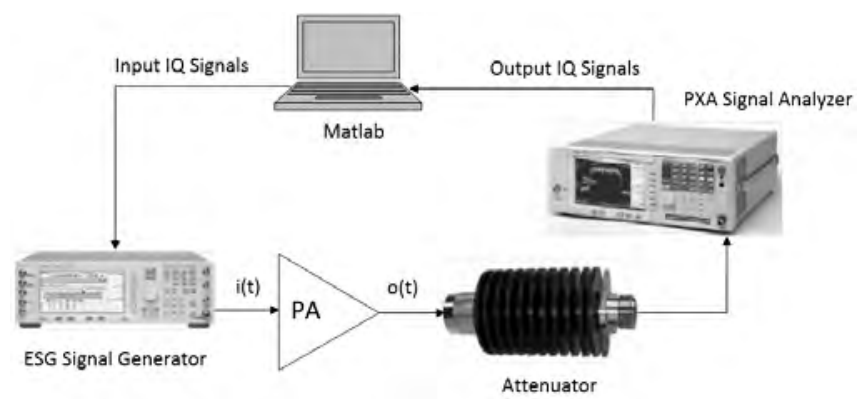

Figure 7. Scheme for data capture.

The input data must be as representative as possible, in order to cover all possible inputs [16]. In this case we select 200 T1 TETRA wanted signals, each consisting of 18 frames. Each frame is composed of three blocks. Frames 1 to 17 are composed by the same normal burst, while frame 18 is composed by a synchronization burst. The total size of the input signal is 18360 bits. The generation and structure of the T1 signal is shown in [18].

The data set to be collected will be the output of the power amplifier using T1 signal as input, at the following different working condition ranges:

- Frequency: from $806 \mathrm{MHz}$ to $870 \mathrm{MHz}$

- Supply voltage: from 2.7 to $4.8 \mathrm{~V}$

- Temperature: from $-30{ }^{\circ} \mathrm{C}$ to $60^{\circ} \mathrm{C}$

The changes in the PA behavior due to variations in frequency, supply voltage and temperature along these ranges make necessary a suitable data collection covering them, therefore allowing an accurate network training, so that it can be capable to conditioning all the input signals. However, in order to reduce the system complexity, a suitable datasets selection must be carried out by means of a properly sampling. In our case, selected values are:

- Frequency: 806, 816, 827, 838, 847, 859, $870 \mathrm{MHz}$

- Battery voltage: $2.7,3.0,3.3,3.6,3.9,4.2,4.5,4.8 \mathrm{~V}$

- Temperature: $-30,25,60^{\circ} \mathrm{C}$

The more input data are used for training (more samples along the whole three ranges), the better the neural network will work for input signals along the frequency, supply voltage and temperature ranges. However, it makes more difficult the convergence to an accurate network configuration. As will be seen, to avoid this we have split the full problem in three simpler ones (one for frequency, one for supply voltage, and one for temperature), thus reducing the total complexity. Firstly (Section IV), selecting a neural 
network architecture to compensate the PA non-linearities at nominal working conditions, next (Section V) compensating the rest of undesired effects.

\section{B. Neural network configuration}

As the goal of this work is to embed a power amplifier linearization system based on an ANN into a low-cost lowsize TETRA handheld, a tradeoff between efficiency, system complexity and size must be reached. It is thus necessary to select the minimum size of the neural network that allows to meet ACP TETRA requirements.

The ANN development was performed using the Matlab Neural Network Toolbox [20]. In order to optimize the training process many schemes have been tested [16]:

Training functions:

- Scaled conjugate gradient backpropagation (SCG)

- Gradient descent with momentum and adaptive learning rate backpropagation (GDX)

- Levenberg-Marquardt backpropagation (LM)

- Bayesian regularization backpropagation (BR)

Transfer functions:

- Hyperbolic tangent sigmoid transfer function (Tanh)

- Linear transfer function (Linear)

- Log-sigmoid transfer function (Logistic)

Performance estimation functions:

- Mean squared normalized error performance function (MSE)

- Sum squared error performance function (SSE)

- Mean absolute error performance function (MAE)

\section{Training and its objective}

As can be seen in Figure 8A, if we consider that the PA transfer function is $A * h(t)$, where $A$ is the constant gain and $h(t)$ represents the non-linearity, the RF output signal is given by:

$$
o(t)=A * h(t) * I(t)
$$

The objective of the training process (Figure 8B) is to calculate the neural network weight set that compensates the effect of $h(t)$, so that for an input o(t) the network provides a signal output the closest to $A * i(t)$, or put another way, the transfer function of the neural network $\mathrm{n}(\mathrm{t})$ must be as similar as possible to $h^{-1}(t)$ (Figure $8 \mathrm{C}$ ).

To achieve this goal, we use the different data sets consisting of phase and quadrature signals previously collected. These data sets (Figure 9) are composed by the output signals from the non-linearized PA $(o(t))$, and the ideally linear PA output signals $(A * i(t))$.

The result obtained from this training process will be the weights of the neural network that better fit to the objective $n(t)=h^{-1}(t)$, therefore minimizing the training error.

\section{Verify generalization ability}

Once the network has been properly configured, minimizing the training error, its generalization ability is verified using 100 different new TETRA patterns [18], not used in the training process: PN9 (9 bits Pseudorandom Number Sequence, PNS), PN11 (11 bits PNS), PN15 (15 bits PNS), PN20 (20 bits PNS), PN23 (23 bits PNS), SCH
(Synchronization Channel Sequence) and TCH (Traffic Channel Sequence).
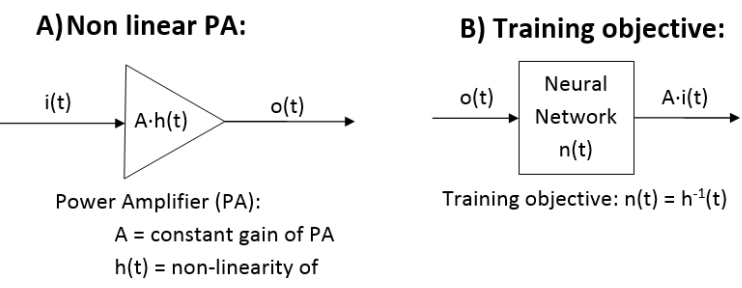

C) When NN is applied to a signal, the non-linearity is corrected:

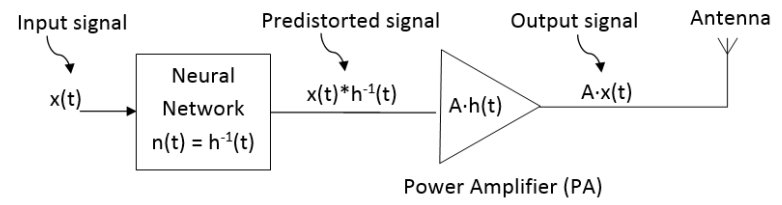

Figure 8. Training objective of the ANN.

\section{NEURAL NETWORK DEVELOPMENT UNDER NOMINAL WORKING CONDITIONS}

Prior to implementing the ANN into the TETRA handheld, it is necessary to verify that the selected architecture meets the previously defined requirements. For this purpose, the predistorted IQ signals are generated using Matlab, and are sent to the power amplifier through the Vector Signal Generator E4438C, which can be configured as an arbitrary generator, providing modulated RF signals from the corresponding IQ signals.

Some different ANN configurations have been tested in order to select the most suitable architecture that minimizes the ACP. The results presented in this Section are average values from 10 different training cases.

Table II shows the effect of varying the training function when a single hidden layer consisting of 20 neurons is used. As Table II shows, the simpler first order algorithms SCG and GDX are not able to provide an ACP value standardcompliant, while both the Levenberg-Marquardt and Bayesian regularization backpropagation do. Otherwise, the additional complexity of the BR algorithm compared to the classical LM does not improve the results obtained.

Table III shows the results obtained using different performance functions applied to a neural network with the aforementioned configuration, trained using the LevenbergMarquardt (LM) algorithm [16].

Table IV shows the effect of varying the transfer function of both the hidden and the output layer in the same network using LM training and mean square error (MSE) performance function. In order to a more general analysis, it has been tested neural networks using different transfer functions in each layer. As expected, network architectures using linear functions in the hidden processors provide worst results, while those architectures where the hyperbolic tangent is the hidden output function provide the best results.

Finally, table V shows the different tested architectures and their corresponding results when the LM training function, MSE performance function and the hyperbolic tangent (hidden layer) and linear (output layer) transfer functions are used. 
TABLE II

ACP VS TRAINING FUNCTION. EXPERIMENTAL CONDITIONS: TEMPERATURE $=25^{\circ} \mathrm{C} ;$ FREQUENCY $=838 \mathrm{MHZ} ;$ VSUPPLY $=3.3 \mathrm{~V} ; \mathrm{VCONTROL}=2.65 \mathrm{~V}$

\begin{tabular}{|c|c|}
\hline Training function & $\mathrm{ACP}\left(\mathrm{P}_{\text {out }}=30 \mathrm{dBm}\right)$ \\
\hline SCG & $45 \mathrm{dBc}$ \\
\hline GDX & $34 \mathrm{dBc}$ \\
\hline LM & $\mathbf{5 7} \mathbf{~ d B c}$ \\
\hline BR & $55.5 \mathrm{dBc}$ \\
\hline
\end{tabular}

TABLE III.

ACP vs PERFormanCE FunCTION. EXPERIMENTAL CONDITIONS: TEMPERATURE $=25^{\circ} \mathrm{C} ;$ FREQUENCY $=838 \mathrm{MHZ} ;$ VSUPPLY $=3.3 \mathrm{~V}$; VCONTROL $=2.65 \mathrm{~V}$.

\begin{tabular}{|c|c|}
\hline Performance function & $\mathrm{ACP}\left(\mathrm{P}_{\text {out }}=30 \mathrm{dBm}\right)$ \\
\hline MSE & $\mathbf{5 7 ~ d B c}$ \\
\hline SSE & $56.8 \mathrm{dBc}$ \\
\hline MAE & $52 \mathrm{dBc}$ \\
\hline
\end{tabular}

TABLE IV.

ACP VS TRANSFER FUNCTION. EXPERIMENTAL CONDITIONS: TEMPERATURE $=25^{\circ} \mathrm{C} ;$ FREQUENCY $=838 \mathrm{MHZ} ;$ VSUPPLY $=3.3 \mathrm{~V}$; VCONTROL $=2.65 \mathrm{~V}$.

\begin{tabular}{|c|c|c|}
\hline \multicolumn{2}{|c|}{ Transfer functions (hidden / output layers) } & $\mathrm{ACP}\left(\mathrm{P}_{\text {out }}=30 \mathrm{dBm}\right)$ \\
\hline Linear & Linear & $47 \mathrm{dBc}$ \\
\hline Logistic & Linear & $52 \mathrm{dBc}$ \\
\hline Tanh & Linear & $57 \mathrm{dBc}$ \\
\hline Linear & Logistic & $32 \mathrm{dBc}$ \\
\hline Linear & Tanh & $34 \mathrm{dBc}$ \\
\hline Logistic & Tanh & $56 \mathrm{dBc}$ \\
\hline Tanh & Tanh & $56.5 \mathrm{dBc}$ \\
\hline
\end{tabular}

TABLE $\mathrm{V}$

ACP VS NUMBER OF HIDDEN LAYERS - NEURONS. EXPERIMENTAL CONDITIONS: TEMPERATURE $=25^{\circ} \mathrm{C} ;$ FREQUENCY $=838 \mathrm{MHZ} ;$ VSUPPLY $=$ $3.3 \mathrm{~V} ; \mathrm{VCONTROL}=2.65 \mathrm{~V}$

\begin{tabular}{|c|c|}
\hline Number of hidden layers and neurons & ACP $\left(\mathrm{P}_{\text {out }}=30 \mathrm{dBm}\right)$ \\
\hline $1-5$ & $49 \mathrm{dBc}$ \\
\hline $1-10$ & $54 \mathrm{dBc}$ \\
\hline $1-15$ & $55.5 \mathrm{dBc}$ \\
\hline $1-16$ & $55.8 \mathrm{dBc}$ \\
\hline $1-17$ & $56 \mathrm{dBc}$ \\
\hline $1-18$ & $56.4 \mathrm{dBc}$ \\
\hline $1-19$ & $56.6 \mathrm{dBc}$ \\
\hline $\mathbf{1 ~ - 2 0}$ & $\mathbf{5 7 ~ d B c}$ \\
\hline $1-30$ & $57.2 \mathrm{dBc}$ \\
\hline $2-20(1$ st hidden $), 10\left(2^{\text {nd }}\right)$ & $57.3 \mathrm{dBc}$ \\
\hline
\end{tabular}

Because the ACP limit in TETRA standard is $55 \mathrm{dBc}$, it is advisable to select an architecture giving a wider value, thus compensating possible tolerances in the amplifier. In addition, it must be noted that the ACP values are given in logarithmic $\mathrm{dBc}$ scale, so that a difference of $1.5 \mathrm{dBc}$ (as is the case in the 1-15 and 1-20 architectures, see Table V) represents a linear factor of about 1.4.

According to the results shown in Tables II to V, an ANN compliant with the aforementioned requirements will consist of one hidden layer with 20 processors working with a hyperbolic tangent output function, linear function in the output layer, using the Levenberg-Marquardt algorithm in the training process with MSE performance function. The resultant neural network is showed in Figure 9.

The number of weights of the final network will be 102, what is fully assumable allowing to introduce the neural network in the DSP of the handheld.

The Levenberg-Marquardt algorithm updates the weights $(w)$ of the neural network during the training, regarding the next equation and in order to minimize the performance function (training error):

$$
w_{K+1}=w_{K}-\left[J^{T} J+\mu I\right]^{-1} J^{T} e
$$

where $J$ is the Jacobian matrix that contains first derivatives of the network errors with respect to the weights and biases, $I$ is the identity matrix, and $e$ is a vector of network errors (MSE in this case).

The scalar value of $\mu$ is variable. The term $\mu$ is decreased after each successful step (MSE reduction) and is increased only when a tentative step would increase the MSE value. In this way, the performance function is always reduced at each iteration of the algorithm.

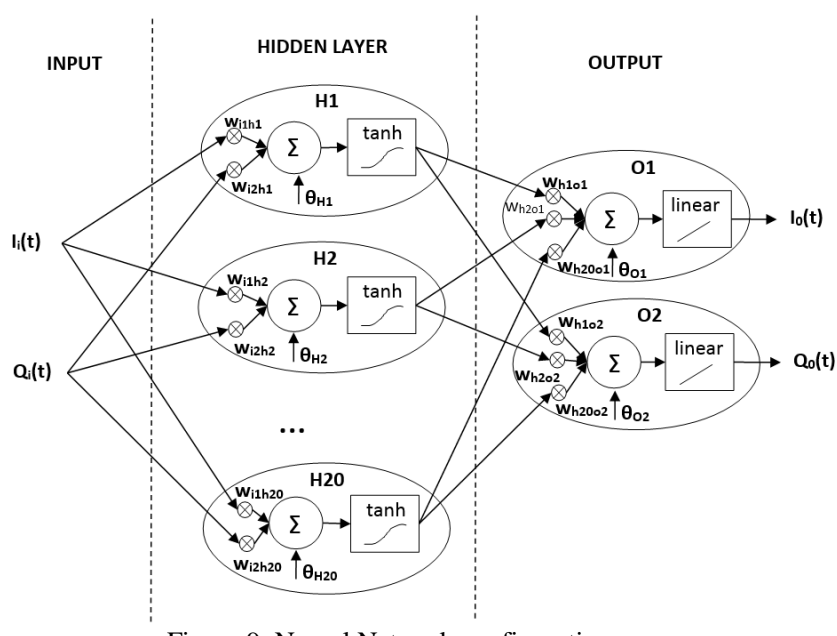

Figure 9. Neural Network configuration.

The gradient can be computed as:

$$
\Delta=J^{T} e
$$

The evolution of the gradient and $\mu$ over time can be seen in figure 10 .

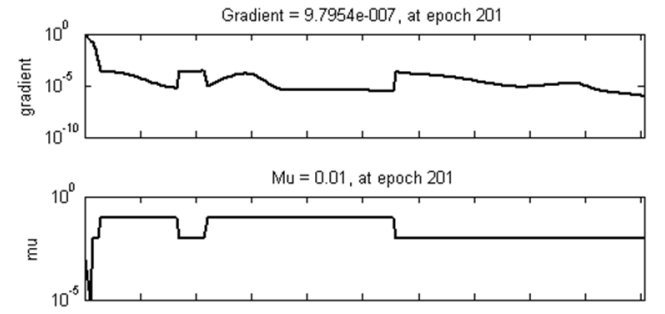

Figure 10. Gradient and $\mu$ evolution over time for a 1-20-1 ANN configuration

During the training process, the network performance evolves according to Figure 11. The best training performance obtained with these conditions was $2.18 \cdot 10^{-4}$, with a minimum gradient of $9.8 \cdot 10^{-7}$. The data used for training is composed by $160 \mathrm{~T} 1$ signals (the $80 \%$ dataset), while network performance test is calculated using the remaining $40 \mathrm{~T} 1$ signals ( $20 \%$ of the dataset).

Figure 12 shows the regression plot, comparing the real output of the network with the desired output (target). In some occasions a reduced MSE value can be obtained having numerous very poorly adjusted training examples (provided the rest have a much lower error), leading to a bad adjustment. This figure is of particular relevance, showing that the low MSE achieved corresponds to a good match for the full data 
set.

Under these conditions the achieved ACP is $57 \mathrm{dBc}$ (Table VI), meeting the TETRA standard limit $(55 \mathrm{dBc})$ [18]. In this way, the ACP of the power amplifier achieves $13 \mathrm{~dB}$ improvement, compared to the amplifier without a linearization technique (see Section II).

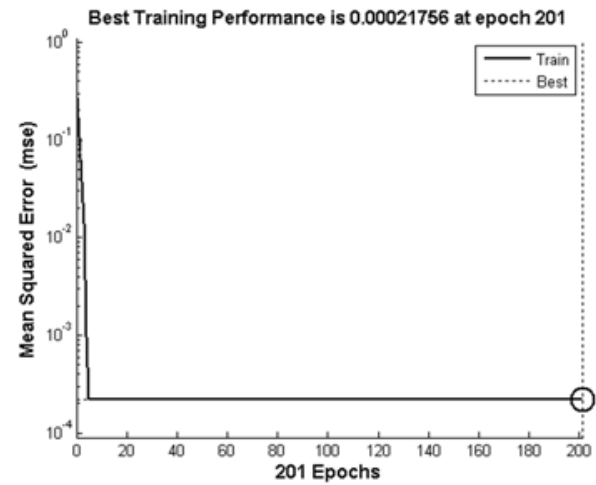

Figure 11. Performance over time for a 1-20-1 ANN. Best MSE value is obtained after 200 iterations.

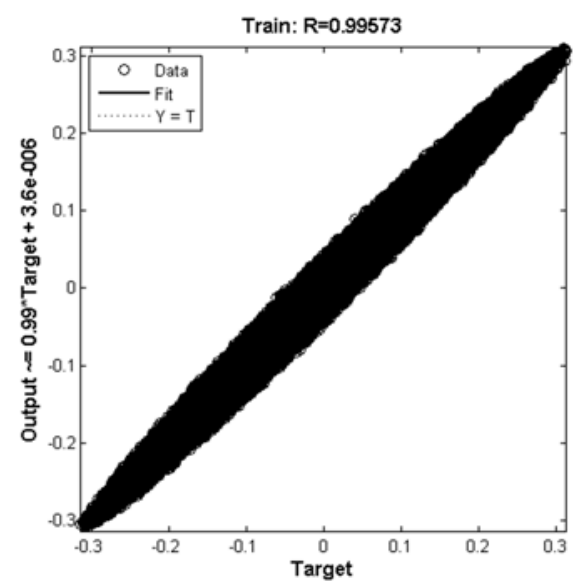

Figure 12. Regression plot after network convergence (maximum allowed MSE reached).

TABLE VI.

ACP AND EVM ACHIEVED FOR DIFFERENT PATTERNS

\begin{tabular}{|c|c|c|c|c|c|c|c|c|}
\hline & T1 & PN9 & PN11 & PN15 & PN20 & PN23 & SCH & TCH \\
\hline $\begin{array}{c}\text { EVM } \\
(\%)\end{array}$ & 2.3 & 2.2 & 2.2 & 2.2 & 2.2 & 2.2 & 2.3 & 2.3 \\
\hline $\begin{array}{c}\text { ACP } \\
(\mathrm{dBc})\end{array}$ & 57 & 57.2 & 57.2 & 57.2 & 57.1 & 57.1 & 57 & 57 \\
\hline
\end{tabular}

\section{V.COMPENSATION TECHNIQUES FOR CHANGES IN WORKING CONDITIONS}

The Cartesian method has a feedback loop that allows to correct the non-linearity of the PA even when the external conditions change. However, our solution, in order to reduce costs, does not have any feedback loop and therefore when transmission frequency (f), supply voltage (Vsupply) or operation temperature $(\mathrm{T})$ change, the power amplifier alters its transfer function, thus reducing the efficiency of the proposed system, therefore reducing the ACP improvement. These effects are shown in Tables VII to IX, applying the obtained architecture to the validation dataset previously generated. In order to consider those effects, it is possible to add the corresponding inputs to a larger linearization ANN. However, this approach can dramatically increase both size and computational complexity of the neural network, thus hindering its implementation in a low-cost electronic processor or field programmable gate array, and therefore moving away from the main purpose of this work.

TABLE VII

ACP VS FREQUENCY. EXPERIMENTAL CONDITIONS: TEMPERATURE $=25^{\circ} \mathrm{C}$

VSUPPLY $=3.3 \mathrm{~V} ;$ OUTPUT POWER $=30 \mathrm{dBm} ; \mathrm{VCONTROL}=2.65 \mathrm{~V}$

\begin{tabular}{|c|c|c|}
\hline Frequency & $\begin{array}{c}\text { ACP with ANN linearization, without } \\
\text { frequency effect compensation }\end{array}$ & $\begin{array}{c}\text { ACP TETRA } \\
\text { standard limit }\end{array}$ \\
\hline $806 \mathrm{MHz}$ & $49.5 \mathrm{dBc}$ & $55 \mathrm{dBc}$ \\
\hline $838 \mathrm{MHz}$ & $57 \mathrm{dBc}$ & $55 \mathrm{dBc}$ \\
\hline $870 \mathrm{MHz}$ & $54 \mathrm{dBc}$ & $55 \mathrm{dBc}$ \\
\hline
\end{tabular}

TABLE VIII.

ACP vS SUPPLY VOLTAGE EXPERIMENTAL CONDITIONS: TEMPERATURE $=$ $25^{\circ} \mathrm{C}$; FREQUENCY $=838 \mathrm{MHZ} ;$ OUTPUT POWER $=30 \mathrm{dBm} ; \mathrm{VCONTROL}=$ $2.65 \mathrm{~V}$.

\begin{tabular}{|c|c|c|}
\hline Vsupply & $\begin{array}{c}\text { ACP with ANN linearization, without } \\
\text { voltage effect compensation }\end{array}$ & $\begin{array}{c}\text { ACP TETRA } \\
\text { standard limit }\end{array}$ \\
\hline $2.7 \mathrm{~V}$ & $42 \mathrm{dBc}$ & $55 \mathrm{dBc}$ \\
\hline $3 \mathrm{~V}$ & $47 \mathrm{dBc}$ & $55 \mathrm{dBc}$ \\
\hline $3.3 \mathrm{~V}$ & $57 \mathrm{dBc}$ & $55 \mathrm{dBc}$ \\
\hline $3.6 \mathrm{~V}$ & $47 \mathrm{dBc}$ & $55 \mathrm{dBc}$ \\
\hline $3.9 \mathrm{~V}$ & $42 \mathrm{dBc}$ & $55 \mathrm{dBc}$ \\
\hline $4.2 \mathrm{~V}$ & $41 \mathrm{dBc}$ & $55 \mathrm{dBc}$ \\
\hline $4.5 \mathrm{~V}$ & $38 \mathrm{dBc}$ & $55 \mathrm{dBc}$ \\
\hline $4.8 \mathrm{~V}$ & $35 \mathrm{dBc}$ & $55 \mathrm{dBc}$ \\
\hline
\end{tabular}

TABLE IX.

ACP VS TEMPERATURE. EXPERIMENTAL CONDITIONS: FREQUENCY = $838 \mathrm{MHZ} ;$ VSUPPLY $=3.3 \mathrm{~V} ;$ OUTPUT POWER $=30 \mathrm{dBm} ; \mathrm{VCONTROL}=2.65$ $\mathrm{V}$

\begin{tabular}{|c|c|c|}
\hline Temperature & $\begin{array}{c}\text { ACP with ANN linearization, } \\
\text { without temperature effect } \\
\text { compensation }\end{array}$ & $\begin{array}{c}\text { ACP TETRA } \\
\text { standard limit }\end{array}$ \\
\hline$-30^{\circ} \mathrm{C}$ & $44 \mathrm{dBc}$ & $45 \mathrm{dBc}$ \\
\hline $25^{\circ} \mathrm{C}$ & $57 \mathrm{dBc}$ & $55 \mathrm{dBc}$ \\
\hline $60^{\circ} \mathrm{C}$ & $53 \mathrm{dBc}$ & $45 \mathrm{dBc}$ \\
\hline
\end{tabular}

A. Frequency effect compensation

It is possible to define three transmission frequency ranges (Figure 13), in order to compensate the frequency effect. Then, for each range of frequency, a different ANN is trained, thus obtaining different weight values, while maintaining the same architecture.

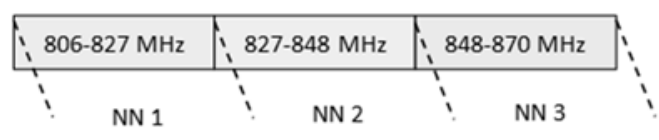

Figure 13. Frequency ranges.

This suggests the existence of three different neural networks with the same structure but different coefficients depending on the frequency used. This frequency segmentation avoids the use of additional inputs, and therefore reduces the computational complexity of the global solution at a low increase of use of data storage resources. Table X and Figure 14 show the ACP results for the full frequency range.

TABLE X.

ACP AFTER FREQUENCY COMPENSATION. EXPERIMENTAL CONDITIONS: TEMPERATURE $=25^{\circ} \mathrm{C} ;$ VSUPPLY $=3.3 \mathrm{~V} ;$ OUTPUT POWER $=30 \mathrm{dBm}$; VCONTROL $=2.65 \mathrm{~V}$

\begin{tabular}{|c|c|}
\hline Frequency & ACP with / without ANN linearization \\
\hline $806-827 \mathrm{MHz}$ & $55 \mathrm{dBc} / 42 \mathrm{dBc}$ \\
\hline $827-848 \mathrm{MHz}$ & $57 \mathrm{dBc} / 44 \mathrm{dBc}$ \\
\hline $848-870 \mathrm{MHz}$ & $56 \mathrm{dBc} / 43 \mathrm{dBc}$ \\
\hline
\end{tabular}


As can be seen, the ACP obtained is slightly better in the central sub-band than in the lower and upper ones, mainly due to the inherent linearity of the power amplifier in this frequency range.

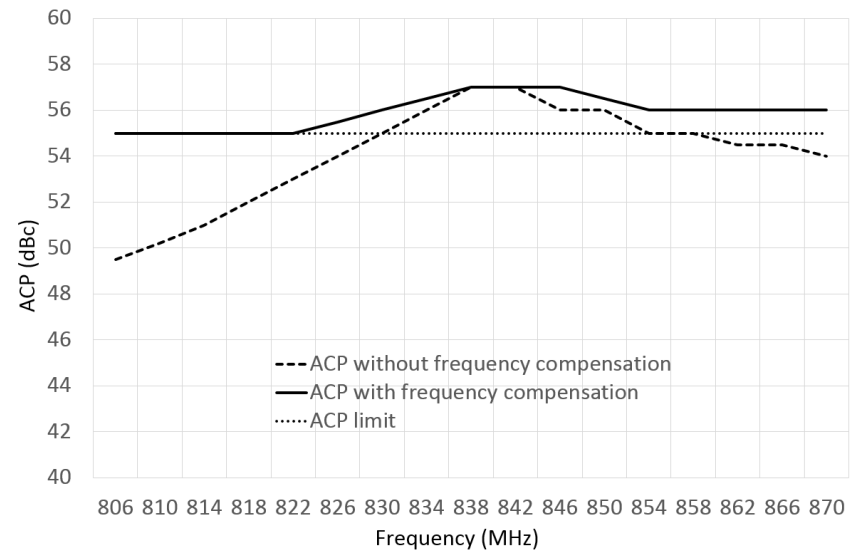

Figure 14. ACP after ANN linearization: before (dash line) and after (continuous line) frequency compensation.

\section{B. Voltage effect compensation}

The effect of supply voltage variations can be compensated by modifying the input power of the power amplifier according to changes in the supply voltage. This input power of the PA can be set by controlling the Vg signal in Figure 15. Table XI shows the input power variations required and the corresponding ACP obtained at the output.

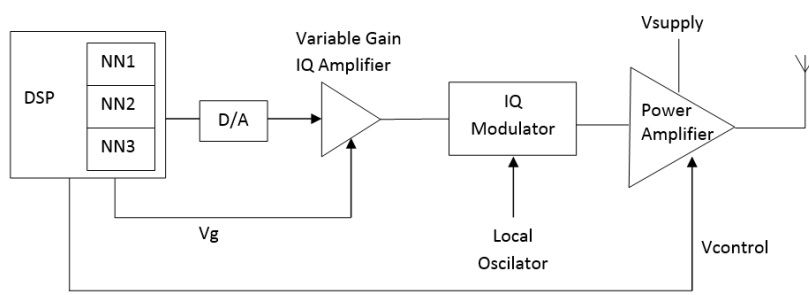

Figure 15. Block diagram of the linearization solution.

TABLE XI.

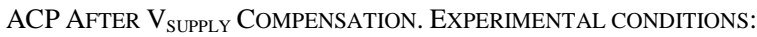
TEMPERATURE $=25^{\circ} \mathrm{C} ;$ FREQUENCY $=838 \mathrm{MHz} ;$ VCONTROL $=2.65 \mathrm{~V}$

\begin{tabular}{|c|c|c|c|}
\hline Vsupply & $\begin{array}{c}\text { Input power } \\
\mathrm{P}_{\text {in }}(\mathrm{Vg})\end{array}$ & $\begin{array}{c}\text { Output power } \\
\mathrm{P}_{\text {out }}\end{array}$ & $\begin{array}{c}\mathrm{ACP} \text { with } \\
\mathrm{Vg}=\mathrm{f}\left(\mathrm{V}_{\text {supply }}\right)\end{array}$ \\
\hline $2.7 \mathrm{~V}$ & $-4.4 \mathrm{dBm}$ & $28.3 \mathrm{dBm}$ & $57.5 \mathrm{dBc}$ \\
\hline $3 \mathrm{~V}$ & $-3.6 \mathrm{dBm}$ & $29.2 \mathrm{dBm}$ & $57.5 \mathrm{dBc}$ \\
\hline $3.3 \mathrm{~V}$ & $-2.6 \mathrm{dBm}$ & $30.1 \mathrm{dBm}$ & $57 \mathrm{dBc}$ \\
\hline $3.6 \mathrm{~V}$ & $-1.9 \mathrm{dBm}$ & $30.9 \mathrm{dBm}$ & $57 \mathrm{dBc}$ \\
\hline $3.9 \mathrm{~V}$ & $-1.2 \mathrm{dBm}$ & $31.6 \mathrm{dBm}$ & $56 \mathrm{dBc}$ \\
\hline $4.2 \mathrm{~V}$ & $-0.3 \mathrm{dBm}$ & $32.0 \mathrm{dBm}$ & $56 \mathrm{dBc}$ \\
\hline $4.5 \mathrm{~V}$ & $0.4 \mathrm{dBm}$ & $32.7 \mathrm{dBm}$ & $55 \mathrm{dBc}$ \\
\hline $4.8 \mathrm{~V}$ & $1.1 \mathrm{dBm}$ & $33.1 \mathrm{dBm}$ & $55 \mathrm{dBc}$ \\
\hline
\end{tabular}

As can be seen in Table XI, the output power varies when the input power is varied. However, the output power keeps meeting the limits of the TETRA standard [18]. Results for the whole supply voltage range are shown in Figure 16.

This solution requires a continuous supply voltage monitoring in order to properly modify the input power. However, the handheld permanently monitors the supply voltage to determine the battery power level and its operating life; hence, no additional computing requirements are needed and computational complexity is reduced, overcoming in this way the need of increase both the ANN structure and the data set size.



Figure 16. ACP after ANN linearization: before (dash line) and after (continuous line) supply voltage compensation.

\section{Compensation of the temperature effect}

Similarly, temperature effects can be compensated by varying the input power (varying Vg in Figure 15) and the gain control of the amplifier (varying Vcontrol in Figure 15) in order to improve the ACP. Table XII and Figure 17 show the results that were achieved for three different working temperatures.

As in the case of supply voltage compensation, this solution requires a continuous temperature monitoring in order to properly modify the voltages $\mathrm{Vg}$ and Vcontrol. Again, because the handheld permanently monitors the temperature in order to compensate several different features, the proposed solution results in the use of no additional computing or electronic resources.

TABLE XII.

ACP AFTER TEMPERATURE COMPENSATION. EXPERIMENTAL CONDITIONS: VSUPPLY $=3.3 \mathrm{~V}$; FREQUENCY $=838 \mathrm{MHZ}$

\begin{tabular}{|c|c|c|c|c|}
\hline Temperature & $\mathrm{P}_{\text {in }}(\mathrm{Vg})$ & Vcontrol & $\mathrm{P}_{\text {out }}$ & ACP \\
\hline$-30^{\circ} \mathrm{C}$ & $-3.1 \mathrm{dBm}$ & $2.75 \mathrm{~V}$ & $30.7 \mathrm{dBm}$ & $56.2 \mathrm{dBc}$ \\
\hline $25^{\circ} \mathrm{C}$ & $-2.6 \mathrm{dBm}$ & $2.65 \mathrm{~V}$ & $30.1 \mathrm{dBm}$ & $57 \mathrm{dBc}$ \\
\hline $60^{\circ} \mathrm{C}$ & $-2 \mathrm{dBm}$ & $2.65 \mathrm{~V}$ & $30.1 \mathrm{dBm}$ & $57 \mathrm{dBc}$ \\
\hline
\end{tabular}



Figure 17. ACP after ANN linearization: before (dash line) and after (continuous line) temperature compensation. 


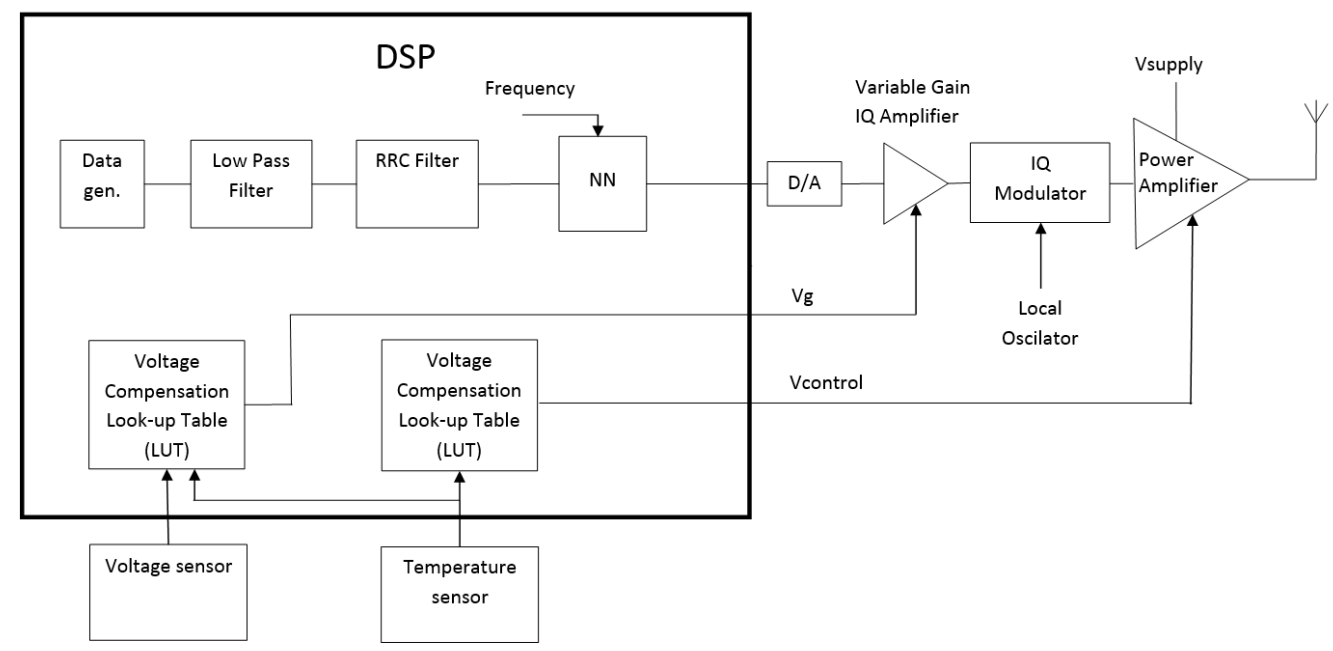

Figure 18. Global solution scheme for the power amplifier linearization by using a neural network.

After considering the aforementioned proposed compensation techniques, Figure 18 presents the complete scheme that has been used to increase the linear range of the power amplifier by means of an ANN, including the compensation of frequency, temperature and supply voltage variations.

Due to the frequency, supply voltage and temperature compensation techniques, the training of the neural network is carried out only during the manufacturing process of the handheld. This greatly reduces the complexity, allowing to simplify both the hardware (a feedback loop is not required) and the software (it is not necessary to recalculate the neural network coefficients), and making feasible the introduction of the neural network in the handheld.

\section{IMPLEMENTATION OF THE NEURAL NETWORK IN A LOW-COST TETRA HANDHELD}

Once the neural network has been trained and validated, it is possible to study its implementation in the standard digital signal processor (DSP) included in the TETRA handheld that has been selected. Other issues have been reported in the literature referring to ANNs implemented in a DSP [22] [25].

In particular, the OMAP5910 dual core processor of Texas Instruments is mounted in this handheld. This processor includes a dual MAC (multiply-accumulate) TMS320C55x DSP architecture. This DSP performs the following functions:

- It generates the TETRA frame

- It applies the predistortion to the TETRA frame by using the ANN

- It selects the suitable ANN architecture and modifies the gain parameters according to the frequency, supply voltage and temperature values

Processing data with an ANN adds a time delay in the output signal. In particular, for the proposed architecture using 20 neurons in the hidden layer and employing the TMS320C55x DSP at 144MHz, the number of clock cycles to calculate the ANN output is around 25000, which corresponds to a time delay of no more than $175 \mu \mathrm{s}$, fully compatible with the timing requirements of the TETRA standard [18].
Figure 19 shows the signal spectrum at the input and output of the power amplifier with and without ANN linearization. The ACP improvement applying the ANN linearization system is $13 \mathrm{~dB}$. Measurements were obtained using an Agilent N9030A PXA Signal Analyzer.

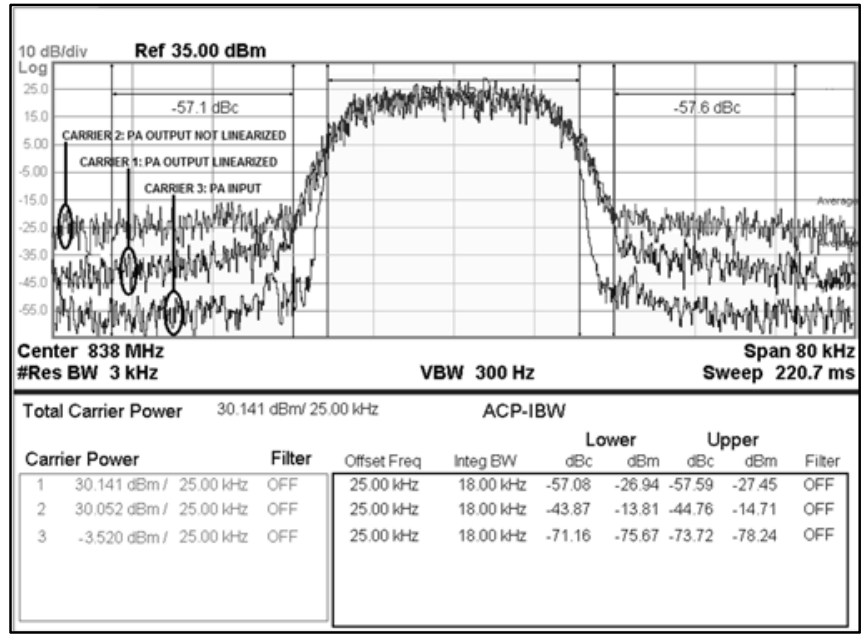

Figure 19. Power amplifier input and output spectrum with and without ANN linearization.

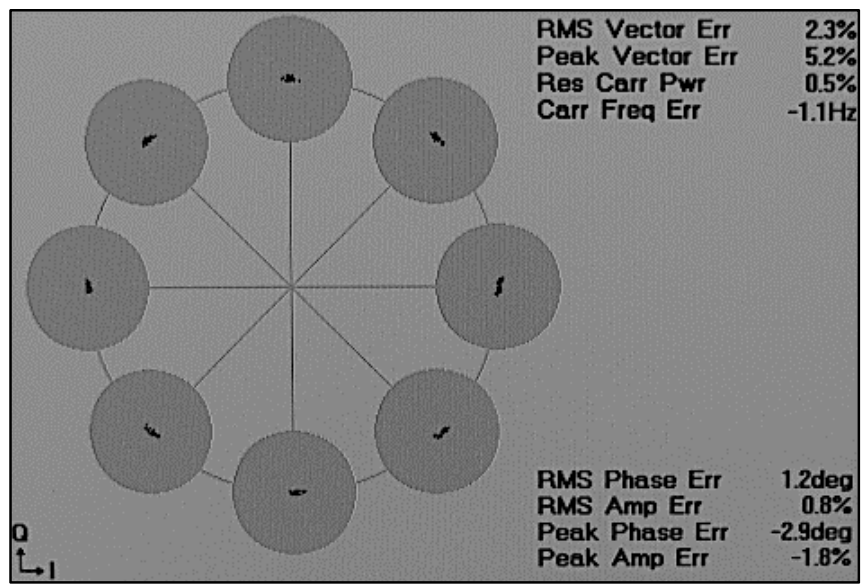

Figure 20. Constellation and EVM after applying the neural network.

Figure 20 shows the EVM (Error Vector Magnitude) [21] of the output signal of the amplifier, obtained by applying the 
ANN linearization. As it can be seen in the constellation diagram, both magnitude and phase errors are complying with the requirements of the TETRA standard [18], which means that the ANN does not increase the in-band distortion of the amplifier. Measurements were obtained using an Aeroflex IFR 2310 TETRA Signal Analyzer.

Table XIII shows the results obtained using the method proposed in this paper, compared to the Cartesian Feedback Loop method implemented in the original handheld, and without linearization method.

TABLE XIII.

COMPARISON BETWEEN CARTESIAN AND ANN LINEARIZATION METHOD.

\begin{tabular}{|c|c|c|c|}
\hline & $\begin{array}{c}\text { Without } \\
\text { Linearization }\end{array}$ & $\begin{array}{c}\text { Cartesian } \\
\text { Feedback Loop }\end{array}$ & $\begin{array}{c}\text { Proposed Neural } \\
\text { Network }\end{array}$ \\
\hline EVM & $2.2 \%$ & $2.4 \%$ & $2.3 \%$ \\
\hline ACP & $44 \mathrm{dBc}$ & $57.2 \mathrm{dBc}$ & $57 \mathrm{dBc}$ \\
\hline
\end{tabular}

As can be seen, the proposed method provides the same performance than the original Cartesian Feedback Loop method, but greatly decreasing the complexity.

\section{CONCLUSIONS}

This paper demonstrates the feasibility of applying an artificial neural network to increase the power transmission efficiency in a low-cost professional RF communications handheld, based on TETRA standard and able to transmit 1 watt in the $806-870 \mathrm{MHz}$ frequency band.

The simple architecture of the ANN required for this task allows its implementation in the low cost Digital Signal Processor (DSP) embedded in the handheld at no significant resource cost. In addition, it is possible to establish a strategy of dividing the predistortion process in different sub-tasks by selecting the ANN parameters according to the frequency band. Furthermore, the effects of supply voltage and temperature can be compensated by varying the amplifier gain with the information provided by the defined LUTs (Look-up Tables).

A first look to the advantages of the new proposed solution using ANN, compared to the traditional Cartesian feedback loop, can be shown by comparing Figures 21 and 22. In the original TETRA handheld block diagram (Figure 21), the DSP is responsible for generating the TETRA frame before its linearization using the Cartesian feedback loop. The Cartesian feedback loop is implemented on an ASIC. If the ANN system is applied instead of the original Cartesian feedback loop (Figure 22), the use of an ASIC to implement the linearization algorithm and the need of a RF feedback path are no longer required, thus leading to an important reduction of the Printed Circuit Board (PCB) area and a 10\% reduction of the cost of the terminal, as well as eliminating the reliance on possible ASIC stock variations, obsolescence or unexpected cost increase. Although the ANN predistortion method requires continuous adjustment $(\mathrm{Vg}$ and Vcontrol in Figure 18), this adjustment is made automatically by the algorithm implemented in the DSP without any additional hardware cost, while the continuous adjustment made for the Cartesian loop requires additional hardware to implement the feedback loop. A resume of pros and contras between Cartesian feedback loop and the proposed ANN predistortion method for RF power amplifiers linearization in terminals is shown in Table XIV. The only contras that the proposed method presents with respect to the Cartesian Loop are the requirement of an initial training of the neural network, and the need for two analog outputs of the DSP. However, neither is relevant, since the initial training is only carried out during the manufacturing process of the terminal using external instrumentation, and the need for an additional analog output is easily obtainable with the DSP.

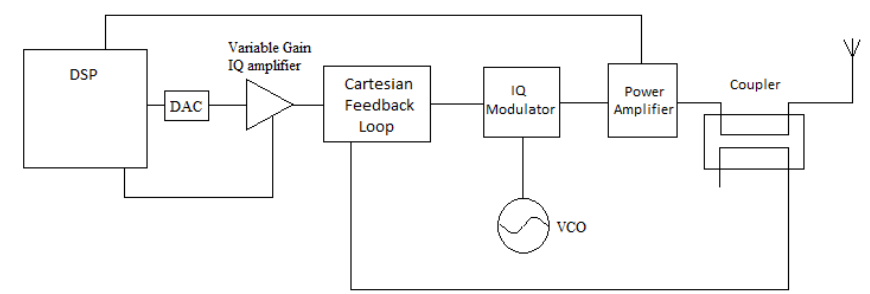

Figure 21. Original transmitter system including Cartesian Feedback loop predistortion.

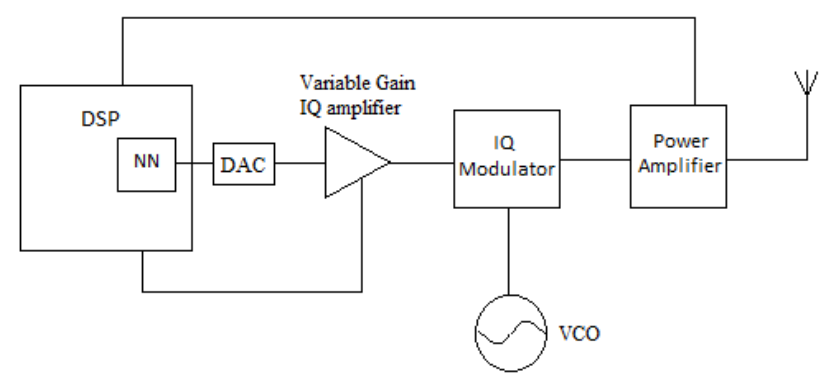

Figure 22. New transmitter system including ANN predistortion.

Unlike previous works that apply complex ANNs to linearize RF power amplifiers [7]-[13] requiring additional computing hardware as FPGAs or powerful DSPs (thus orienting this solutions to static infrastructures), this work performs the practical implementation of a simple ANN into the DSP of a radio terminal, and the compensation of possible changes in working conditions such as frequency, temperature and supply voltage, therefore allowing to simplify and eliminate parts of hardware that until now were necessary. This will have repercussions in a reduction of cost and size of the terminals. The cost reduction due to the implementation of the neural network in the DSP of the handheld can be increased with an additional $17 \%$ by using the RF5110G amplifier that can be supplied with only one $3.7 \mathrm{~V}$ lithium cell, instead of the normally used transistors that need two lithium cells $(7.4 \mathrm{~V})$. This means that the proposed linearization technique can reduce the handheld cost in more than a $25 \%$.

TABLE XIV

COMPARISON BETWEEN CARTESIAN AND PROPOSED ANN METHOD.

\begin{tabular}{|c|l|l|}
\hline $\begin{array}{c}\text { Cartesian } \\
\text { Feedback } \\
\text { Loop }\end{array}$ & $\begin{array}{l}\text { Not initial learning } \\
\text { required. } \\
\text { Only one DSP analog } \\
\text { output required (Vg). }\end{array}$ & $\begin{array}{l}\text { Feedback loop required. } \\
\text { Higher economic cost. } \\
\text { Greater PCB area required. } \\
\text { Possible need of an ASIC. }\end{array}$ \\
\hline ANN & $\begin{array}{l}\text { Less economic cost. } \\
\text { linearization }\end{array}$ & $\begin{array}{l}\text { Initial learning required. } \\
\text { Two DSP analog outputs } \\
\text { Not feedback loop } \\
\text { required. }\end{array}$ \\
\hline
\end{tabular}

For future works, it will be interesting to apply this neural network method: 
- In the 300-400MHz Tetra band.

- With amplifiers with other technologies such as LDMOS or GaN.

- With wide band modulations such as LTE.

This work is a real alternative to the Cartesian Feedback Loop method used in low cost terminals for linearizing the RF power amplifier. In addition, though this work has been focused on TETRA standard, it can be extrapolated easily to any mobile phone terminal, which makes this work a tremendously attractive solution for the telecommunications industry due to its design simplicity, which allows an easy implementation into the DSP of any low cost terminal, so that can be applied to other digital modulation standards like GSM, UMTS, LTE, etc., simplifying the hardware of the devices and hence reducing the cost of terminals, while maintaining their performance and meeting the standards of each modulation.

\section{ACKNOWLEDGMENTS}

This work has been partially funded by TEC2015-65750R (MINECO/FEDER, UE) and by the company Teltronic.

The authors would like to thank the company Teltronic for the collaboration in the tests that have been described in this work.

\section{REFERENCES}

[1] Pipilos, S, Papananos, Y., Naskas, N., Zervakis, M., Jakob Jongsma, Gschier, T., Wilson, N., Gibbins, J., Carter, B., Dann, G., "A transmitter IC for TETRA systems based on a Cartesian feedback loop linearization technique", IEEE Journal of Solid State Circuits, vol. 40, no. 3, pp. 707-718, Mar. 2005.

[2] Gokceoglu, A.; Ghadam, A.; Valkama, M., "Steady-State Performance Analysis and Step-Size Selection for LMS-Adaptive Wideband Feedforward Power Amplifier Linearizer", IEEE Transactions on Signal Processing, vol. 60, no. 1, pp. 82-99, Jan. 2012.

[3] Haoran Yu, Kamal El-Sankary and Ezz I. El-Masry, "Distortion Analysis Using Volterra Series and Linearization Technique of NanoScale Bulk-Driven CMOS RF Amplifier" IEEE Transactions on Circuits and Systems I: regular papers, vol. 62, no. 1, pp. 19-28, Jan. 2015.

[4] Morgan, D.R. ; Zhengxiang Ma ; Jaehyeong Kim ; Zierdt, M.G. ; Pastalan, J., "A Generalized Memory Polynomial Model for Digital Predistortion of RF Power Amplifiers", IEEE Transactions on Signal Processing, vol.54, no. 10, pp 3852-3860, Oct. 2006.

[5] Gilabert, P.L.; Montoro, G.; Bertran, E., "On the Wiener and Hammerstein models for power amplifier predistortion", Microwave Conference Proceedings, 2005. APMC 2005. Asia-Pacific Conference Proceedings, vol. 2.

[6] Bo Ai ; Zhi-Xing Yang ; Chang-Yong Pan ; Shi-gang Tang ; Tao-tao Zhang, "Analysis on LUT Based Predistortion Method for HPA with Memory", IEEE Transactions on Broadcasting, vol. 53, no. 1, Mar. 2007.

[7] Zhan Su, Janusz Kolbusz, and Bogdan M. Wilamowski, "Linearization of Bipolar Amplifiers Based on Neural-Network Training Algorithm", IEEE Transactions on Industrial Electronics, vol. 63, no. 6, pp. 37373744, June 2016

[8] S. Boumaiza and F. Mkadem, "Wideband RF power amplifier predistortion using real-valued time-delay neural networks," in Proceedings 39th European Microwave Conference, October 2009, pp. 1449-1452.

[9] M. Rawat, K. Rawat, and F. M. Ghannouchi, "Adaptive Digital Predistortion of Wireless Power Amplifiers/Transmitters Using Dynamic Real-Valued Focused Time-Delay Line Neural Networks," IEEE Trans. Microwave Theory and Techniques, vol. 58, no. 1, pp. 95104, Jan. 2010.

[10] F. Mkadem, M. B. Ayed, S. Boumaiza, J. Wood, and P. Aaen, "Behavioral modeling and digital predistortion of power amplifiers with memory using two hidden layers artificial neural networks," in IEEE MTT-S Int. Microw. Symp. Dig., 2010, pp. 656-659.
[11] F. Mkadem, S. Boumaiza, "Physically Inspired Neural Network Model for RF Power Amplifier Behavioral Modeling and Digital Predistortion," in IEEE Trans. Microwave Theory and Techniques, vol. 59, no. 4, pp. 913-923, Apr. 2011

[12] M. Rawat and F. M. Ghannouchi, "Distributed Spatiotemporal Neural Network for Nonlinear Dynamic Transmitter Modeling and Adaptive Digital Predistortion," IEEE Trans. Instrumentation and Measurement, vol. 61, no. 3, pp. 595-608, Mar. 2012.

[13] Naskas N., Papananos Y., "Neural-Network-Based Adaptive Baseband Predistortion Method for RF Power Amplifiers", IEEE Trans. Circuits and Systems-II, vol. 51, no. 11, pp. 619-623, Nov. 2004.

[14] Jiménez, V.P.G. ; Jabrane, Y. ; Armada, A.G. ; Said, B.A.E. ; Ouahman, A.A., "High Power Amplifier Pre-Distorter Based on Neural-Fuzzy Systems for OFDM Signals", IEEE Transactions on Broadcasting, vol. 57, no. 1, pp. 149 - 158, Mar. 2011.

[15] Moritz, R. ; Leung, H. ; Xinping Huang, "Nonlinear Compensation for High Power Amplifiers using Genetic Programming", IEEE International Symposium on Circuits and Systems, 2007. ISCAS 2007, pp. $2323-2326$.

[16] S. Haykin, Neural Networks and Learning Machines, 3rd ed. Upper Saddle River, NJ: Prentice-Hall, 2009.

[17] P.B. Kenington, High-Linearity RF Amplifier Design. Boston, MA: Artech House, 2000.

[18] ETSI EN300 394-1 V3.1.1 (2007-11). Terrestrial Trunked Radio (TETRA); Conformance testing specification; Part1: Radio.

[19] RFMD Datasheet DS110914: RF5110G, 3V general purpose GSM power amplifier.

[20] Hudson M., Hagan M.T., Demuth H.B., "Matlab. Neural Network Toolbox. User's Guide", ed. R2014b, Oct. 2014.

[21] Fuqin Xiong, Digital Modulation Techniques, 2nd ed. Artech House, 2006.

[22] Kim N, Kehtarnavaz N, Yeary MB, Thornton S., "DSP-based hierarchical neural network modulation signal classification", IEEE Transactions on Neural Networks, vol. 14, no. 5, pp. 1065-1071, Sept. 2003.

[23] Yang F., Paindavoine M., "Implementation of an RBF neural network on embedded systems: real-time face tracking and identity verification", IEEE Transactions on Neural Networks, vol. 14, no. 5, pp. 1162-1175, Sept. 2003.

[24] Seul Jung, Sung Su Kim, "Hardware Implementation of a Real-Time Neural Network Controller with a DSP and an FPGA for Nonlinear Systems", IEEE Transactions on Industrial Electronics, vol. 54, no. 1, pp. 265-271, Feb. 2007.

[25] Muller, U.A. ; Gunzinger, A. ; Guggenbuhl, Walter, "Fast neural net simulation with a DSP processor array", IEEE Transactions on Neural Networks, vol. 6, no. 1, pp. 203-213, Jan. 1995.

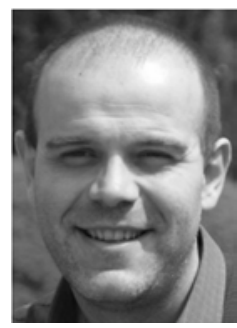

Raúl Gracia was born in Zaragoza, Spain. $\mathrm{He}$ received the B.Sc. degree in Telecommunications Engineering from the University of Zaragoza, Zaragoza, Spain, in 1999.

Currently, he is working towards the Ph.D. degree at the Electronic Engineering and Communications Department, University of Zaragoza, while working at Teltronic. His research interests include neural networks application for radio frequency systems, radio communications and microwaves.



Nicolás Medrano (M'96) was born in Zaragoza, Spain. He received the B.Sc. and Ph.D. degrees in Physics from the University of Zaragoza, Zaragoza, Spain, in 1989 and 1998, respectively.

He is currently an Associate Professor of Electronics with the Faculty of Physics and a Member of the Group of Electronic Design, Aragon Institute of Engineering Research, University of Zaragoza. His current research interests include adaptive signal processing, integrated sensor interfaces, wireless sensor networks, and intelligent instrumentation. 